\title{
Fast Algorithms for Complete Linkage Clustering*
}

\author{
D. Krznaric and C. Levcopoulos \\ Department of Computer Science, Lund University, \\ Box 118, S-221 00 Lund, Sweden \\ drago@dna.lth.se \\ christos@dna.lth.se
}

\begin{abstract}
It is shown that the complete linkage clustering of $n$ points can be computed in $O\left(n \log ^{2} n\right)$ time. Furthermore, it is shown that the complete linkage clustering can be approximated within an arbitrarily small constant factor in $O(n \log n)$ time.
\end{abstract}

\section{Introduction}

Given a set $S$ of $n$ objects, the complete linkage (c-link) method produces a hierarchy of clusters as follows. Initially, each object in $S$ constitutes a cluster. Then, as long as there is more than one cluster, a closest pair of clusters is merged into a single cluster. The c-link distance of two clusters $C$ and $C^{\prime}, \delta\left(C, C^{\prime}\right)$, is defined as $\max \left\{|x y|: x \in C\right.$ and $\left.y \in C^{\prime}\right\}$, where $|x y|$ denotes the distance (dissimilarity) between objects $x$ and $y$.

Hierarchical clustering algorithms are important for structuring and interpreting data in domains such as biology, medicine, geographical information systems, and image processing [6]. Among the different methods for producing a hierarchy of clusters, the c-link clustering is one of the most well known, and has thus been used for applications [1], [5], [6].

The obvious algorithm for computing the c-link clustering takes cubic time. Using priority queues, Day and Edelsbrunner [3] showed that it can be obtained in $O\left(n^{2} \log n\right)$ time. A quadratic-time algorithm was described by Murtagh [13]. Recently, Křrivánek [11] developed a quadratic-time algorithm based on the $(a, b)$-tree data structure. (These three algorithms also work for some other clustering methods.) A quadratic-time algorithm that uses linear space was proposed by Defays [4]; however, it only approximates the hierarchy since its output depends on a certain insertion order [2]. Parallel algorithms

\footnotetext{
* A preliminary version appeared at the International Symposium on Algorithms and Computation, 1995.
} 
for the c-link clustering have also been developed [10], [12], but asymptotically the total work was still at least quadratic.

We consider the case where $S$ consists of $n$ distinct points in the plane and distances are measured according to the Euclidean metric. In this setting, we show that the c-link clustering can be computed in $O\left(n \log ^{2} n\right)$ time and linear space. Furthermore, we show that an $\varepsilon$-approximation can be computed in $O\left(n \log n+n \log ^{2}(1 / \varepsilon)\right)$ time (actually $O\left(n \log ^{2}(1 / \varepsilon)\right)$ time suffices if the Voronoi diagram is given). By an $\varepsilon$-approximation we mean a hierarchy that can be produced like a c-link hierarchy, except that the following holds at each merging: if $P$ is the pair of clusters next merged and $P^{\prime}$ is a closest pair of clusters, then $\delta(P) \leq(1+\varepsilon) \delta\left(P^{\prime}\right)$. The techniques presented in this paper can also be generalized to other types of clusterings.

The paper is organized as follows. In Section 2 we define a hierarchy of well-separated clusters, which is easy to compute and useful in order to organize the algorithm in a hierarchical fashion. In that section we also state a lemma that is crucial for our approach, giving an upper bound on the number of clusters that can overlap with a small region. An outline of the algorithm is given in Section 3, where we describe how linear-time behavior can be achieved by merging "locally closest" pairs of clusters. In Section 4 we explain how our algorithm computes all c-link distances in $O\left(n \log ^{2} n\right)$ time, by using dynamization techniques for merging farthest point Voronoi diagrams. Finally, in Section 5 we present our algorithm for computing an $\varepsilon$-approximation of a c-link hierarchy.

\section{Preliminaries}

We use the hierarchical clustering method from [9] to decompose $S$ into subsets for which the c-link clustering can be computed separately. Define the rectangular diameter of a point set $D$, abbreviated $\operatorname{rdiam}(D)$, as the diameter of the smallest axis-aligned rectangle that contains all points of $D$.

Definition 2.1. A subset $D$ of $S$ is a 2-cluster if the distance between any point of $D$ and any point of $S-D$ is greater than $2 \cdot \operatorname{rdiam}(D)$ or $D$ equals $S$.

It is easy to show that any two different 2-clusters are either disjoint or one of them is a proper subset of the other. This property causes the 2-clusters to form a unique hierarchy, defined as follows:

Definition 2.2. The 2-cluster tree of $S$ is a rooted tree whose nodes correspond to distinct 2-clusters, where the root corresponds to $S$ and the external nodes to single points of $S$. Let $a$ be any internal node and let $A$ be its corresponding 2-cluster. Then the children of $a$ correspond to each 2-cluster $C$ such that $C \subset A$ and there is no 2-cluster $B$ such that $C \subset B \subset A$.

In the continuation, by a 2-cluster we also refer to its corresponding node of the 2cluster tree and vice versa. A simple algorithm that computes the 2-cluster tree in $O(n)$ time from a Euclidean minimum spanning tree of $S$ was presented in [9]. As a by-product 
of that algorithm, for each 2-cluster $D$ we receive the minimum distance between any point of $D$ and any point of $S-D$. The following is another property about 2-clusters that we use:

Fact 2.3 (Lemma 3.3 in [9]). For any 2-cluster $D$ with $m \geq 2$ children, let $d$ and $d^{\prime}$ be the minimum and maximum distance, respectively, between any two points belonging to distinct children of $D$. Then $d^{\prime}<2^{2 m-3} d$.

We define an $\varepsilon$-approximation of a c-link hierarchy as follows:

Definition 2.4. An $\varepsilon$-procedure produces a hierarchy of clusters by repeatedly merging two clusters in such a way that the following holds at each merging: if $P$ is the pair of clusters next merged and $P^{\prime}$ is a closest pair of clusters, then $\delta(P) \leq(1+\varepsilon) \delta\left(P^{\prime}\right)$. We say that a hierarchy of clusters is an $\varepsilon$-approximation of a c-link hierarchy if it can be produced by an $\varepsilon$-procedure.

The algorithm in the next section merges a pair $\left(C, C^{\prime}\right)$ of clusters only if it is a locally closest pair, in the sense that no other cluster is within c-link distance $<\delta\left(C, C^{\prime}\right)$ from $C$ or $C^{\prime}$. The main reason for this approach is that we can efficiently check whether a pair is locally closest, because there are only a constant number of other clusters in its vicinity. This is formalized in the following lemma:

Lemma 2.5. For every $\varepsilon$-procedure with $\varepsilon \leq 0.1$, the following holds after each merging: if the c-link distance of each pair of clusters is greater than l, then no disk with radius $O(l)$ contains more than a constant number of clusters.

Proof. Let $d$ be the distance of a closest pair of points in the input $S$. We denote by $t_{i}$ the time just after the $\varepsilon$-procedure makes the last merging for which the following holds: after this merging, the c-link distance of a closest pair of clusters is $\leq 2^{8 i} d$. We say that a square overlaps a cluster if it contains at least one point of the cluster. All squares used in this proof have sides parallel to the coordinate axes.

The proof is by induction on $i$. The induction hypothesis is as follows: at time $t_{i}$, for some arbitrary $i$, no square with side length $2^{8 i} d$ overlaps more than $2^{31}$ clusters. (Our intuition is that the true constant is not larger than, say, 50.) It is easy to see that no square with side length $d$ overlaps more than four clusters (recall, initially each cluster corresponds to a single point in $S$ ). Thus the statement is true for $i=0$. Let $s$ be any square with side length $2^{8 i+8} d$. It remains to show that $s$ does not overlap more than $2^{31}$ clusters at time $t_{i+1}$.

Let $s^{\prime}$ be the square with side length $2^{8 i+10} d$ that is concentric with $s$. Before time $t_{i+1}$, we merge a pair of clusters only if the c-link distance of the pair is $\leq 1.1 \cdot 2^{8 i+8} d$. Hence, no cluster with diameter $>1.1 \cdot 2^{8 i+8} d$ exists at time $t_{i+1}$ (the diameter of a cluster is the distance between its two points that lie farthest apart). Therefore, at any time before $t_{i+1}$, each cluster that $s$ overlaps is completely internal to $s^{\prime}$.

The $2^{30}$ squares that partition $s^{\prime}$ into subsquares with sides of length $2^{8 i-5} d$ are called triggers. Define the enclosing disk of a cluster as the smallest disk containing all points of the cluster. Now, suppose that there are at time $t_{i}$ two clusters $C$ and $C^{\prime}$ such that 
their enclosing disks are centered in the same trigger. Since $C$ has diameter $\leq 1.1 \cdot 2^{8 i} d$, the enclosing disk of $C$ has diameter $\leq(2 / \sqrt{3}) 1.1 \cdot 2^{8 i} d<1.28 \cdot 2^{8 i} d$ (consider an equilateral triangle inscribed in a disk). The same holds for $C^{\prime}$. Hence, $\delta\left(C, C^{\prime}\right)<$ $\sqrt{2} \cdot 2^{8 i-5} d+1.28 \cdot 2^{8 i} d<1.33 \cdot 2^{8 i} d$. This means that $C$ or $C^{\prime}$, say $C$, will be merged with either $C^{\prime}$ or some cluster $C^{\prime \prime}$ such that $\delta\left(C, C^{\prime \prime}\right)<1.1 \cdot 1.33 \cdot 2^{8 i} d<1.47 \cdot 2^{8 i} d$.

Let $m$ be the number of clusters that are completely internal to $s^{\prime}$ at time $t_{i}$ (from now on we only consider clusters that are completely internal to $s^{\prime}$, since $s$ can only overlap such clusters at time $\left.t_{i+1}\right)$. By the observation in the previous paragraph, at least $m-2^{30}$ clusters will participate in a merging, in such a way that each new cluster resulting from these mergings has diameter $<1.47 \cdot 2^{8 i} d$. The number of clusters that remain after these mergings is at most $2^{30}+\left(m-2^{30}\right) / 2$, where each remaining cluster has an enclosing disk with diameter $<(2 / \sqrt{3}) 1.47 \cdot 2^{8 i} d<1.7 \cdot 2^{8 i} d$. We can repeat the same scenario for the remaining clusters. After having done that we are left with at most $2^{30}+\left(m-2^{30}\right) / 4$ clusters, each cluster having diameter $<1.1\left(\sqrt{2} \cdot 2^{8 i-5} d+1.7 \cdot 2^{8 i} d\right)<1.92 \cdot 2^{8 i} d$. Indeed, we can repeat the scenario as long as we afterwards are left with at least one pair of clusters of c-link distance $\leq 2^{8 i+8} d$, that is, as long as we can assure that time $t_{i+1}$ is not reached (we may assume that there are after each repetition sufficiently many clusters left for the next repetition to work).

Now, if we repeat the scenario $k$ times, we are left with at most

$$
2^{30}+\frac{m-2^{30}}{2^{k}}<2^{30}+\frac{m}{2^{k}}
$$

clusters, and among these clusters there are at least two between which the c-link distance is

$$
\leq 2^{8 i} d\left(\frac{1.1 \cdot 2}{\sqrt{3}}\right)^{k+1}+\sqrt{2} \cdot 2^{8 i-5} d \sum_{j=0}^{k}\left(\frac{1.1 \cdot 2}{\sqrt{3}}\right)^{j},
$$

which is easily shown to be less than $2^{8 i+8} d$ for $k \leq 21$. But since $s^{\prime}$ can be partitioned into $2^{20}$ squares with sides of length $2^{8 i} d$, we have by our induction hypothesis that $m \leq 2^{20+31}=2^{51}$. Hence, at time $t_{i+1}$, the number of clusters that $s$ overlaps is at most

$$
2^{30}+\frac{2^{51}}{2^{21}}=2^{31}
$$

\section{An Outline of the Algorithm}

\subsection{Processing Each 2-Cluster Separately and in Phases}

It is easy to see that each 2-cluster is also a cluster in any c-link hierarchy. Thus we can compute a c-link hierarchy by a post-order traversal of the 2-cluster tree. In this way it suffices to consider the following problem: given the children of a 2-cluster $D$, cluster the children of $D$ according to the c-link method. We cluster the children of $D$ in a sequence $p_{0}, p_{1}, \ldots$ of phases. More precisely, let $l$ be the distance of a closest pair of points that belong to distinct children of $D$. Then the objective of a phase $p_{i}$ is to merge every pair $\left(C, C^{\prime}\right)$ of clusters such that $l_{i} \leq \delta\left(C, C^{\prime}\right)<2 l_{i}$, where $l_{i}$ equals $2^{i} l$. By the parameter of phase $p_{i}$ we will mean $l_{i}$. 
Now, consider a child $D^{\prime}$ of $D$. Let $l^{\prime}$ be the minimum distance between any point in $D^{\prime}$ and any point in $S-D^{\prime}$. Clearly, there is no other cluster within c-link distance $<l^{\prime}$ from $D^{\prime}$, so $D^{\prime}$ can be ignored until we reach a phase at which we merge pairs of c-link distance $\geq l^{\prime}$. Therefore, if $l_{i} \leq l^{\prime}<2 l_{i}, D^{\prime}$ is assigned to phase $p_{i}$. In this way we assign each child of $D$ to a phase, and each child is kept idle until we reach the phase which it is assigned to. By Fact 2.3, the time used to assign the children to various phases is linear in the number of children.

\subsection{Computing a Phase $p_{i}$}

The clusters that remain at the end of phase $p_{i-1}$ are passed on to the next phase (each such cluster is composed of children of $D$ that were assigned to phases preceding $p_{i}$ ). Let $L$ be a list consisting of these clusters and those clusters (children of $D$ ) that have been assigned to phase $p_{i}$. For a cluster $C$, define $N(C)$ as any set that includes every other cluster in $L$ within c-link distance $<2 l_{i}$ from $C$, but does not include any cluster $C^{\prime}$ such that $\delta\left(C, C^{\prime}\right)$ is greater than, say, $10 l_{i}$. By Lemma 2.5, $N(C)$ contains a constant number of clusters. Therefore, we can find such a set $N(C)$ in constant time by using the range searching technique developed in [8]. That technique builds basically on the construction of a threaded quadtree for $S$, which can be done in $O(n)$ time given the Voronoi diagram of $S$ (see [8] for more details).

We use a graph $G$ to explain how phase $p_{i}$ works, defined as follows. The vertices of $G$ correspond to the clusters in $L$. In addition, for each pair $\left(C, C^{\prime}\right)$ of vertices (clusters) there is an edge connecting $C$ and $C^{\prime}$ if and only if $\delta\left(C, C^{\prime}\right)<2 l_{i}$. The length of an edge $\left(C, C^{\prime}\right)$ is defined to be equal to $\delta\left(C, C^{\prime}\right)$. Clearly, to compute which edges that are to be incident to a vertex $C$, it suffices to consider the constant number of clusters in $N(C)$. Now, starting with an empty stack, repeat the following four steps until $G$ has no edges:

1. Pop $e$ from the stack (if the stack is empty, let $e$ be any edge of $G$ ).

2. Let $e^{\prime}$ be a shortest edge adjacent to $e$ (if no edge is adjacent to $e$, go to Step 4).

3. If $e^{\prime}$ is shorter than $e$, push $e$ and $e^{\prime}$ onto the stack (in this order) and go to Step 1.

4. Merge the clusters connected by $e$ into a single cluster. This means that $e$ is contracted, that is, $G$ is updated as follows:

(a) Add a new vertex $C_{e}$ to $G$.

(b) For each edge $\left(C, C^{\prime}\right)$ such that $C^{\prime}$ is an endpoint of $e$, add $\left(C, C_{e}\right)$ to $G$ if $\delta\left(C, C_{e}\right)<2 l_{i}$ (and if $\left(C, C_{e}\right)$ does not already exist).

(c) If the stack is nonempty, pop and discard the edge on top (this edge is adjacent to $e$ ).

(d) Delete the endpoints of $e$ and all edges adjacent to $e$ from $G$.

When an edge $e$ is contracted at Step 4, there is no shorter edge adjacent to $e$. It is easy to realize that this means that we would eventually contract $e$ even if we would contract edges in increasing order according to their lengths. Hence, we merge the same pairs of clusters as the c-link method, although we may merge them in a different order. The vertices (clusters) that remain after the above procedure are passed on to the next phase. 


\subsection{Analysis of the Run Time}

By Lemma 2.5, there is a constant number of edges incident to each vertex of $G$. Moreover, after contracting an edge, we may pop one edge at Step 4(c), and one more at Step 1 when the loop repeats. However, the second edge that we pop is either pushed back on the stack at Step 3 or contracted at Step 4. Consequently, we do not successively pop more than twice without contracting an edge. Hence, if we temporarily ignore the time used for computing c-link distances between clusters, the total time used by phase $p_{i}$ is linearly bounded by the number of clusters in $L$.

Now, let $C$ be a cluster in $L$. Clearly, there is a cluster $C^{\prime}$ within c-link distance $O\left(l_{i}\right)$ from $C$ ( $C$ would otherwise constitute a 2-cluster, which is not possible because of the way in which we assign the children of $D$ to various phases). Therefore, within a constant number of phases after $p_{i}, C$ or $C^{\prime}$ must participate in a merging. Let us associate $C$ with the first such merging. In this way, each merging is associated with clusters during at most a constant number of phases. Moreover, at each such phase we associate at most a constant number of clusters to the merging, by Lemma 2.5. Hence, since we perform at most $n-1$ mergings during the whole algorithm, we obtain the following lemma:

Lemma 3.1. Let $S$ be a set of $n$ points in the Euclidean plane. Given the Voronoi diagram of $S$, the above algorithm computes the complete linkage clustering of $S$ in time $O(n)$ plus the time taken to compute c-link distances between clusters.

\section{Computing Complete Linkage Distances}

To efficiently compute c-link distances between clusters, each cluster is kept partitioned into subsets, where each subset has what we call a Voronoi structure. (The ideas in this section bear resemblance to standard dynamization techniques presented in, for example, [14].) The Voronoi structure of a set $V$ of points consists of the following three pieces:

1. the farthest point Voronoi diagram of $V$;

2. a data structure for point location in this Voronoi diagram; and

3. the (at most) four extreme points of $V$ in the $x$ and $y$ direction, which we call xy-extremes.

We can use, for example, Kirkpatrick's [7] data structure for point location, and compute the farthest point Voronoi diagram using the algorithm of Shamos [16]. The Voronoi structure of $V$ can then be constructed in $O(|V| \log |V|)$ time, and, using this Voronoi structure, we can for an arbitrary point $p$ find a point of $V$ that is farthest from $p$ in $O(\log |V|)$ time.

Initially, each point of $S$ is equipped with its trivial Voronoi structure. For a cluster $C$, a subset of $C$ that is equipped with a Voronoi structure is called a Voronoi child (or vorchild for short). The vorchildren of a cluster are kept in a list for which we maintain the following two properties:

(1) the vorchildren appear in the list by increasing order with respect to their cardinalities; and 
(2) the cardinalities of two consecutive vorchildren in the list differ at least by a factor 4 .

Thus the number of vorchildren of a cluster is logarithmic with respect to the cardinality of the cluster.

Now, in order to maintain the above two properties, whenever a pair $\left(C_{1}, C_{2}\right)$ of clusters is merged into a single cluster $C$, we impose the following operations. Let $n_{1}$ and $n_{2}$ be the cardinalities of $C_{1}$ and $C_{2}$, respectively, with $n_{1} \leq n_{2}$. First, each vorchild of $C_{2}$ that has cardinality $\leq 4 n_{1}$ and all vorchildren of $C_{1}$ are merged into a single vorchild $V$ (their Voronoi structures are thrown away and a new Voronoi structure for their union is constructed). Next we create a list $L$ that consists of $V$ behind which we hang on what remains of $C_{2}$ 's list of vorchildren (we remove a vorchild from a list when it participates in a merging). It is easy to show that $|V|<7 n_{1}$, so the vorchildren in $L$ are sorted with respect to their cardinalities. Now, as long as the cardinality of the first (smallest) vorchild in $L$ is larger than a quarter of the second vorchild in $L$, merge these two vorchildren into a single vorchild and insert the newly created vorchild first in $L$. The list $L$ that remains after this procedure constitutes $C$ 's list of vorchildren, and it certainly has the two above mentioned properties.

It remains to describe how the c-link distance of a pair $\left(C_{1}, C_{2}\right)$ of clusters is computed at a phase $p_{i}$. Let $V_{1}$ and $V_{2}$ be two vorchildren of $C_{1}$ and $C_{2}$, respectively. First, compute the rectangular diameter, $\operatorname{rdiam}\left(V_{1} \cup V_{2}\right)$, which was defined in Section 2 . The rectangular diameter is easily obtained in constant time by using the $x y$-extremes of $V_{1}$ and $V_{2}$. If $\operatorname{rdiam}\left(V_{1} \cup V_{2}\right)<l_{i}$ (the parameter of $p_{i}$ ), then also $\delta\left(V_{1}, V_{2}\right)<l_{i}$, and so this pair of vorchildren is not relevant (the c-link distance of $\left(C_{1}, C_{2}\right)$ is $\geq l_{i}$, otherwise they would have been merged at some previous phase). Therefore, only if $\operatorname{rdiam}\left(V_{1} \cup V_{2}\right) \geq l_{i}$, we proceed and compute the c-link distance of $\left(V_{1}, V_{2}\right)$ as follows. We can assume that $\left|V_{1}\right| \leq\left|V_{2}\right|$ (otherwise, swap $V_{1}$ and $V_{2}$ ). Let $v$ be a point in $V_{1}$. Again, if $\operatorname{rdiam}\left(\{v\} \cup V_{2}\right)<l_{i}, v$ is not relevant. Otherwise, we use the Voronoi structure of $V_{2}$ to find a point of $V_{2}$ that lies farthest from $v$ in logarithmic time. By considering each point of $V_{1}$ in this way, we compute the c-link distance of $\left(V_{1}, V_{2}\right)$ (if it is $\left.\geq l_{i}\right)$. Finally, by following this procedure for all pairs of vorchildren, one from $C_{1}$ and the other from $C_{2}$, we obtain the c-link distance of $\left(C_{1}, C_{2}\right)$.

Theorem 4.1. Let $S$ be a set of $n$ points in the Euclidean plane. Then the complete linkage clustering of $S$ can be computed in $O\left(n \log ^{2} n\right)$ time using $O(n)$ space.

Proof. Throughout the proof, by $\log n$ we mean $\left\lceil\log _{2} n\right\rceil$.

Lemma 4.2. The total time used for constructing Voronoi structures is $O\left(n \log ^{2} n\right)$.

Proof. Let $v$ be an arbitrary point in $S$. When $v$ participates in a merging of vorchildren, we can differ between the following two cases:

1. The vorchild that $v$ belongs to after merging has cardinality at least 1.25 times the cardinality of the vorchild that $v$ belonged to before merging. It is easy to show that $v$ can participate in a merging of this type at most $\lceil 3.2 \log n\rceil$ times. 
2. The point $v$ belongs to a cluster that is being merged with a cluster of at least the same cardinality. It is easily seen that $v$ can participate in a merging of this type at most $\log n$ times.

When constructing the Voronoi structure of a set $V$, each point in $V$ contributes $O(\log |V|)$ time. Thus the total time used for computing Voronoi structures is $O\left(n \log ^{2} n\right)$.

Next we note that the total time spent for computing the rectangular diameter of pairs of vorchildren is $O\left(n \log ^{2} n\right)$, because each cluster has at most $\log n$ vorchildren and the complete linkage distance is computed for at most $O(n)$ pairs of clusters (see Section 3.3).

For the sake of analysis, we say that a point $v$ makes a $k$-query on a vorchild $V_{2}$ when we use the point location structure of $V_{2}$ to find a point of $V_{2}$ that lies farthest from $v$ and $V_{2}$ has cardinality in $\left[2^{k}, 2^{k+1}\right)$. Similarly, we say that a point $v$ makes a $k$ estimate on a vorchild $V_{2}$ when we compute $\operatorname{rdiam}\left(\{v\} \cup V_{2}\right)$ and $2^{k} \leq\left|V_{2}\right|<2^{k+1}$ (just before deciding whether $v$ should make a query). To complete the proof of our theorem it suffices to show that if each point uses $O(\log n)$ time to make any $k$-query and $O(1)$ time to make any $k$-estimate, then the total time used by the algorithm is $O\left(n \log ^{2} n\right)$. For this purpose, imagine a bank containing coins, the coins having a value so that $\log n$ coins suffice to make any $k$-query and one coin suffices to make any $k$-estimate. In the remainder, $c$ is a sufficiently large constant (an upper bound on $c$ can be derived from this proof, but we do not explicitly calculate it here).

The bank spends coins as follows. Initially, each point receives $c \log ^{2} n+c \log n$ coins from the bank. At any phase $p_{i}$ with parameter $l_{i}$, whenever a vorchild $V_{1}$ expires by some merging, if a point $v$ is within c-link distance $\leq 10 l_{i}$ from $V_{1}$ and belongs to a vorchild (possibly $V_{1}$ ) with cardinality $\leq 2\left|V_{1}\right|$, then $v$ receives $2 c \log n$ coins from the bank.

Lemma 4.3. The total amount of coins that the bank spends is $O\left(n \log ^{2} n\right)$.

Proof. Let $V_{1}$ be a vorchild that expires by merging at a phase $p_{i}$, and let $n_{1}$ be the cardinality of $V_{1}$. First, recall that a cluster has at most one vorchild of cardinality in $\left[2^{k}, 2^{k+2}\right.$ ) for every integer $k$. Hence, if we consider all vorchildren with cardinality $\leq 2 n_{1}$ that belong to the same cluster, then the total sum of their cardinalities is $<3 n_{1}$. But there are at most $O(1)$ clusters within distance $O\left(l_{i}\right)$ from $V_{1}$, by Lemma 2.5. Consequently, when $V_{1}$ expires it causes the bank to spend a total amount of $O\left(n_{1} \log n\right)$ coins. So each point in $V_{1}$ contributes $O(\log n)$ coins to this amount. But it follows from the proof of Lemma 4.2 that the total sum of cardinalities of all vorchildren that are ever created is $O(n \log n)$. Hence, the total amount of coins that the bank spends is $O\left(n \log ^{2} n\right)$.

Now, let $v$ be an arbitrary point in $S$. It remains to show that $v$ can afford to spend $\log n$ coins whenever it makes a $k$-query and one coin whenever it makes a $k$-estimate. To do this, imagine arrays $Q$ and $E$ with slots $q_{0}, q_{1}, \ldots, q_{\log n-1}$ and $e_{0}, e_{1}, \ldots, e_{\log n-1}$, respectively. The point $v$ spends $\log n$ coins in slot $q_{k}$ whenever it makes a $k$-query, and one coin in $e_{k}$ whenever it makes a $k$-estimate. The $c \log ^{2} n+c \log n$ coins that $v$ received initially from the bank are distributed so that each slot of $Q$ contains $c \log n$ 
coins, whereas each slot of $E$ contains $c$ coins. Whenever $v$ receives $2 c \log n$ coins from the bank, because some vorchild $V_{1}$ (where $V_{1}$ may include $v$ ) in the vicinity of $v$ expires, it distributes the coins as follows: $c$ coins are inserted in each slot of $E$, and, if $2^{k} \leq\left|V_{1}\right|<2^{k+1}, c \log n$ coins are inserted in slot $q_{k}$.

Lemma 4.4. For a sufficiently large constant c, no slot of array $Q$ becomes empty.

Proof. Assume for a contradiction that $v$ inserts $c \log n$ coins in $q_{k}$ at a time $t_{1}$ and that $v$ after that makes $c k$-queries without inserting any coin in $q_{k}$, and let $t_{2}$ be the time when the last of these $c k$-queries occurs. Let us first give a rough overview of the proof. It uses the fact that a cluster can have at most one vorchild on which $v$ can make a $k$-query. So, if $v$ makes $c k$-queries between $t_{1}$ and $t_{2}$, there are about $c$ clusters in the vicinity of $v$, contradicting Lemma 2.5 for a sufficiently large $c$. To make this work, we also need to prove two other things:

(1) if $v$ makes a $k$-query on a vorchild between $t_{1}$ and $t_{2}$, then that vorchild does not expire before time $t_{2}$; and

(2) $v$ only makes a constant number (not depending on $c$ ) of $k$-queries on a particular vorchild.

Let $V^{\prime}$ be any vorchild such that $v$ makes a $k$-query on $V^{\prime}$ between $t_{1}$ and $t_{2}$. In this paragraph we show that $V^{\prime}$ cannot expire by merging before time $t_{2}$. If $v$ becomes a member of a vorchild with cardinality $\geq 2^{k+1}$, then it cannot make a $k$-query again (a point does not make queries on vorchildren that are smaller than its own vorchild). Therefore, $v$ belongs to a vorchild of cardinality $<2^{k+1}\left(\leq 2\left|V^{\prime}\right|\right)$ between $t_{1}$ and $t_{2}$. As our algorithm works, if $v$ makes a $k$-query on $V^{\prime}$ during a phase $p_{i}, v$ is within c-link distance $\leq 10 l_{i}$ from $V^{\prime}$. Hence, if $V^{\prime}$ would expire by merging before time $t_{2}, v$ would at that moment insert $c \log n$ more coins in $q_{k}$, a contradiction.

Next we claim that $v$ can make at most $c^{\prime} k$-queries on $V^{\prime}$, where $c^{\prime}$ is a constant not depending on $c$. If $v$ makes a $k$-query on $V^{\prime}$ during a phase $p_{i}$, then within a constant number of phases after $p_{i}$ we reach a phase $p_{j}$ such that $\operatorname{rdiam}\left(\{v\} \cup V^{\prime}\right)<l_{j}$, which means that $v$ does not make any $k$-query on $V^{\prime}$ after $p_{j}$. So, to prove our claim it suffices to show that $v$ cannot make more than $O(1) k$-queries on $V^{\prime}$ during any phase $p_{i}$. But this follows easily because there are at most $O(1)$ clusters within distance $O\left(l_{i}\right)$ from $v$, by Lemma 2.5, and hence at most $O(1)$ clusters containing $v$ or $V^{\prime}$ can be created during $p_{i}$.

Let $p_{r}$ be the phase that includes time $t_{2}$. In the previous paragraph we showed that $v$ can make at most $c^{\prime} k$-queries on the same vorchild between $t_{1}$ and $t_{2}$. Hence, between $t_{1}$ and $t_{2}, v$ makes $k$-queries on at least $c / c^{\prime}$ different vorchildren, and none of these vorchildren can expire before time $t_{2}$. But each cluster can have at most one vorchild with cardinality in $\left[2^{k}, 2^{k+1}\right)$. Thus, at time $t_{2}$, there are at least $c / c^{\prime}$ clusters within distance $\leq 12 l_{j}$ from $v$, contradicting Lemma 2.5 for a sufficiently large $c$.

It remains to show that our arbitrary point $v$ can spend one coin in slot $e_{k}$ whenever it makes a $k$-estimate. Assume for a contradiction that $v$ inserts $c$ coins in $e_{k}$ at a time $t_{1}$ and that $v$ after that makes $c k$-estimates without inserting any coin in $e_{k}$, and let $t_{2}$ be the time when the last of these $c k$-estimates occurs. Let $V$ be the vorchild that 
contains $v$ immediately after time $t_{1}$. Clearly, $V$ cannot expire by merging before time $t_{2}$, because $v$ would at such a moment insert $c$ more coins in $e_{k}$. Now, suppose that $v$ makes a $k$-estimate on a vorchild $V^{\prime}$ between $t_{1}$ and $t_{2}$. As shown in the second paragraph of the proof of Lemma 4.4, $V^{\prime}$ cannot expire by merging before time $t_{2}$, so both $V$ and $V^{\prime}$ remain until time $t_{2}$. But if $v$ makes a $k$-estimate on $V^{\prime}$ during a phase $p_{i}$, then within a constant number of phases after $p_{i}$ we reach a phase $p_{j}$ such that $\operatorname{rdiam}\left(V \cup V^{\prime}\right)<l_{j}$. Consequently, no point of $V$ makes a $k$-estimate on $V^{\prime}$ after phase $p_{j}$. Let $p_{r}$ be the phase that includes time $t_{2}$. As in the proof of Lemma 4.4, there is a constant $c^{\prime}$ not depending on $c$ such that there are at time $t_{2}$ at least $c / c^{\prime}$ clusters within distance $\leq 12 l_{j}$ from $v$, contradicting Lemma 2.5 for a sufficiently large $c$.

\section{Approximating Complete Linkage Distances}

For each cluster we keep track of its extreme points in $k$ directions ( $k$ is an integer $\geq 4$ and the directions are uniformly chosen so that the angle between consecutive directions equals $2 \pi / k$ ). These so-called $k$-extremes are used in order to approximate c-link distances of pairs of clusters. We define the $k$-distance of two clusters $C$ and $C^{\prime}$, denoted by $\delta_{k}\left(C, C^{\prime}\right)$, as the distance of two $k$-extremes, one from $C$ and the other from $C^{\prime}$, that are farthest apart. (We can see from the proof of the following lemma that we could (for even $k$ ) equally well define the $k$-distance considering only pairs of $k$-extremes that are extreme in opposite directions. This might be preferable for a large $k$.)

Lemma 5.1. For any two clusters $C$ and $C^{\prime}$,

$$
1-\frac{5}{k^{2}}<\frac{\delta_{k}\left(C, C^{\prime}\right)}{\delta\left(C, C^{\prime}\right)} \leq 1
$$

Proof. The upper bound is obvious. To prove the lower bound, let $e$ be a longest straightline segment connecting a point of $C$ with a point of $C^{\prime}$ (so the length of $e$ equals $\left.\delta\left(C, C^{\prime}\right)\right)$. Assume without loss of generality that $e$ is vertical and that its lowermost endpoint belongs to $C$. Further, let $l$ and $l^{\prime}$ be two parallel straight lines passing through the lower and the upper endpoint of $e$, respectively (see Fig. 1). Finally, let $\alpha(\geq 0)$ be the smallest angle between $l$ and the horizontal line passing through the lower endpoint of $e$.

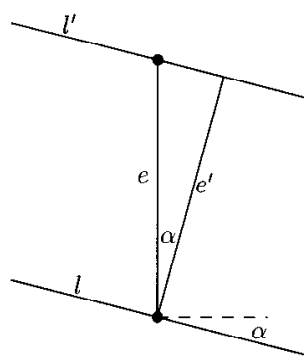

Fig. 1. For the proof of Lemma 5.1. 
Now, suppose that $l$ is chosen so that $\alpha$ is the minimum value for which there is a $k$-extreme of $C^{\prime}$ on or above $l^{\prime}$ and a $k$-extreme of $C$ on or below $l$. Hence, $\alpha \leq \pi / k$ (which is $\leq \pi / 4$ since $k \geq 4$ ). Let $e^{\prime}$ be the straight-line segment perpendicular to $l^{\prime}$ that connects the lower endpoint of $e$ with a point on $l^{\prime}$. Clearly, since there is a $k$-extreme on both sides of the strip bounded by $l$ and $l^{\prime}, \delta_{k}\left(C, C^{\prime}\right) \geq\left|e^{\prime}\right|$. Consider the right-angled triangle bounded by $e, e^{\prime}$, and $l^{\prime}$. It is straightforward to show that the angle between $e^{\prime}$ and $e$ equals $\alpha$. Hence, by traditional trigonometry we have that $\left|e^{\prime}\right| /|e| \geq \cos (\pi / k)$. Using the Maclaurin series, we get that $\cos (\pi / k) \geq 1-(\pi / k)^{2} / 2>1-\overline{5} / k^{2}$, and so $\delta_{k}\left(C, C^{\prime}\right) / \delta\left(C, C^{\prime}\right)>1-5 / k^{2}$.

Lemma 5.2. Using the $k$-distance in the algorithm of Section 3 , we compute an $\varepsilon$ approximation of a complete linkage hierarchy with $\varepsilon<5 /\left(k^{2}-5\right)$.

Proof. Let $D$ be any 2-cluster with at least two children. It is easy to see that $D$ is produced by any $\varepsilon$-procedure for $\varepsilon<1$ (the c-link distance of any two subsets of $D$ is $\leq \operatorname{rdiam}(D)$, whereas the c-link distance between a subset of $D$ and any set disjoint from $D$ is $>2 \cdot \operatorname{rdiam}(D))$. Therefore, in the continuation it suffices to consider the mergings performed by the algorithm when it clusters the children of $D$.

Let $M$ be a list consisting of all pairs of clusters merged by the algorithm, ordered according to their c-link distances (thus not necessarily in the order they were merged by the algorithm). Now, consider the situation after having merged the $i-1$ first pairs in $M$. Let $P$ be the $i$ th pair in $M$ and let $P^{\prime}$ be a closest pair of clusters. It remains to show that $\delta(P) / \delta\left(P^{\prime}\right)<1+5 /\left(k^{2}-5\right)$.

Let $P^{\prime \prime}$ be the first pair in $M$ that contains a cluster of $P^{\prime}\left(P^{\prime}\right.$ and $P^{\prime \prime}$ might be the same pair). Clearly, $\delta(P) \leq \delta\left(P^{\prime \prime}\right)$. We also have that $\delta_{k}\left(P^{\prime \prime}\right) \leq \delta_{k}\left(P^{\prime}\right)$, because we would otherwise merge $P^{\prime}$ instead of $P^{\prime \prime}$. Putting all together and using Lemma 5.1, we get that

$$
\begin{aligned}
\frac{\delta(P)}{\delta\left(P^{\prime}\right)} & \leq \frac{\delta\left(P^{\prime \prime}\right)}{\delta_{k}\left(P^{\prime}\right)}<\frac{\delta_{k}\left(P^{\prime \prime}\right)}{\left(1-5 / k^{2}\right) \delta_{k}\left(P^{\prime}\right)} \\
& \leq \frac{\delta_{k}\left(P^{\prime \prime}\right)}{\left(1-5 / k^{2}\right) \delta_{k}\left(P^{\prime \prime}\right)}=\left(1-\frac{5}{k^{2}}\right)^{-1}=1+\frac{5}{k^{2}-5}
\end{aligned}
$$

From Lemma 5.2 it follows that the statement in Lemma 2.5 holds if $k \geq 8$ ( $\varepsilon$ is then $<0.1$ ). For smaller values on $k$ (between 4 and 7) we can instead rely on the following lemma (note that we in this lemma do not consider an arbitrary $\varepsilon$-procedure):

Lemma 5.3. Using the $k$-distance in the algorithm of Section 3 , at any phase $p_{i}$, no disk of radius $O\left(l_{i}\right)$ contains more than a constant number of clusters (within the 2-cluster being processed).

Proof. The proof is by induction on $i$. We say that a square overlaps a cluster if it contains a $k$-extreme of the cluster (all squares used in this proof have sides parallel to the coordinate axes). The induction hypothesis is as follows: at the beginning of phase $p_{i}$ (before any clusters are merged) no square with side length $l_{i}$ overlaps more than $2 \lambda$ 
clusters, where $\lambda$ is a constant that we specify later. At the beginning of phase $p_{0}$, each cluster corresponds to a child of the 2-cluster being processed, and $l_{0}$ equals the distance of a closest pair of points belonging to distinct children. From this it easily follows that the statement is true for $i=0$. Let $s$ be any square with side length $2 l_{i}$. To complete the proof it suffices to show that $s$ overlaps at most $2 \lambda$ clusters at the beginning of phase $p_{i+1}$.

From the set of clusters that exist at the beginning of phase $p_{i}$ we extract two subsets $H$ and $H^{\prime}$ as follows. The set $H$ consists of every cluster that $s$ overlaps. The set $H^{\prime}$ consists of every cluster that, at the end of phase $p_{i}$, is included in a cluster containing a cluster of $H$. Note that $H \subseteq H^{\prime}$ and that the clusters in $H^{\prime}$ are merged only with each other during phase $p_{i}$. We aim to show that sufficiently many mergings of clusters in $H^{\prime}$ occur during phase $p_{i}$. But first we need some more definitions.

Let $s^{\prime}$ be the square with side length $6 l_{i}$ that is concentric with $s$. It is not hard to see that each cluster in $H^{\prime}$ has all of its $k$-extremes in $s^{\prime}$. Define the triggers as the $r^{2}$ squares that partition $s^{\prime}$ into subsquares with side length $6 l_{i} / r$, where $r=60$. Now, consider a cluster with all its $k$-extremes in $s^{\prime}$. For each $k$-extreme of the cluster we select the trigger in which it is contained, thus selecting $k$ triggers $t_{1}, t_{2}, \ldots, t_{k}$. We say that the cluster is of type $\tau$, where $\tau$ is the unordered $k$-tuple $\left(t_{1}, t_{2}, \ldots, t_{k}\right)$. We are now in position to set the constant $\lambda$, namely, $\lambda$ equals the maximum number of distinct types of clusters. By traditional combinatorics, $\lambda=\left(\begin{array}{c}r^{2}+k-1 \\ k\end{array}\right)$.

Next, define the $k$-diameter of a cluster as the distance of its two $k$-extremes that are farthest apart. It is easily seen that if $C$ and $C^{\prime}$ are any two clusters produced by our algorithm, then the $k$-diameter of $C \cup C^{\prime}$ equals $\delta_{k}\left(C, C^{\prime}\right)$.

Observation 5.4. Let $C$ and $C^{\prime}$ be two clusters with all their k-extremes in s's such that each of them is of type $\tau$ and has $k$-diameter less than $l$. Then the $k$-diameter of $C \cup C^{\prime}$ is less than $l+6 l_{i} \sqrt{2} / r$.

Proof. Since $C$ and $C^{\prime}$ are of the same type, there are $k$ triggers (not necessarily distinct) such that each of them contains two $k$-extremes, one from $C$ and the other from $C^{\prime}$. Let $p$ and $p^{\prime}$ be two $k$-extremes of $C \cup C^{\prime}$ such that the distance between them is maximized. So the $k$-diameter of $C \cup C^{\prime}$ equals $\left|p p^{\prime}\right|$. We can assume that $p$ is a $k$-extreme of $C$ and that $p^{\prime}$ is a $k$-extreme of $C^{\prime}$, because $\left|p p^{\prime}\right|$ would otherwise be $<l$. Let $t_{p}$ be the trigger containing $p$. As mentioned above, $t_{p}$ also contains a $k$-extreme $q^{\prime}$ of $C^{\prime}$, and we know that $\left|p^{\prime} q^{\prime}\right|<l$. But $\left|p q^{\prime}\right|$ is at most the diameter of $t_{p}$, which is equal to $6 l_{i} \sqrt{2} / r$. Hence, by triangle inequality, $\left|p p^{\prime}\right|<l+6 l_{i} \sqrt{2} / r$.

Suppose that there is a subset $T$ of $H^{\prime}$ consisting only of clusters that are of the same type. Let $C$ and $C^{\prime}$ be two clusters in $T$. First we observe that both $C$ and $C^{\prime}$ have $k$-diameter $<l_{i}$, because they were created at some phase before $p_{i}$. By Observation 5.4, the $k$-diameter of $C \cup C^{\prime}$ is $<l_{i}+6 l_{i} \sqrt{2} / r$. Consequently, during phase $p_{i}$ at least one of $C$ and $C^{\prime}$, let us say $C$, will be merged with a cluster $C^{\prime \prime}$ such that $C \cup C^{\prime \prime}$ has $k$-diameter $<l_{i}+6 l_{i} \sqrt{2} / r$ ( $C^{\prime \prime}$ and $C^{\prime}$ might be the same cluster). Thus we realize that each cluster in $T$ except at most one will participate in a merging during phase $p_{i}$, in such a way that the new clusters resulting from these mergings have $k$-diameters $<l_{i}+6 l_{i} \sqrt{2} / r$. 
In the remainder we only consider the clusters in $H^{\prime}$ and those clusters that are created during phase $p_{i}$ by merging clusters in $H^{\prime}$. Let $n^{\prime}$ equal the number of clusters in $H^{\prime}$. As indicated in the previous paragraph, at least $n^{\prime}-\lambda$ clusters will participate in a merging during phase $p_{i}$, and the new clusters resulting from these mergings have $k$-diameters $<l_{i}+6 l_{i} \sqrt{2} / r$. The number of clusters that remain after these mergings is at most $\left(n^{\prime}-\lambda\right) / 2+\lambda$. We can repeat the scenario for the remaining clusters. After having done that we are left with at most $\left(n^{\prime}-\lambda\right) / 2^{2}+\lambda$ clusters, each cluster having $k$-diameter $<l_{i}+2 \cdot 6 l_{i} \sqrt{2} / r$. Indeed, we can repeat the scenario as long as we do not merge two clusters whose union has $k$-diameter $\geq 2 l_{i}$ (we may assume that there are after each repetition sufficiently many clusters left for the next repetition to work).

Now, if we repeat the scenario $j$ times, we are left with at most

$$
\frac{n^{\prime}-\lambda}{2^{j}}+\lambda<\frac{n^{\prime}}{2^{j}}+\lambda
$$

clusters, each cluster having $k$-diameter

$$
<l_{i}+j \cdot 6 l_{i} \sqrt{2} / r
$$

which is $<2 l_{i}$ for $j \leq 7$. But since $s^{\prime}$ can be partitioned into $6^{2}$ subsquares with side length $l_{i}$, we have by our induction hypothesis that $n^{\prime} \leq 6^{2}(2 \lambda)$. Hence, at the end of phase $p_{i}$, the number of clusters that $s$ overlaps is at most

$$
\frac{6^{2}(2 \lambda)}{2^{7}}+\lambda<2 \lambda
$$

which completes the proof.

For two clusters $C$ and $C^{\prime}$ we can compute $\delta_{k}\left(C, C^{\prime}\right)$ in $O(k)$ time by keeping track of the convex hull of the $k$-extremes of each cluster. Indeed, the diameter of a convex hull can be computed in linear time, and two convex hulls can be merged in linear time (see, e.g., Sections 3.3.5 and 4.2.3 in [15]). Hence, the total time used for computing $k$-distances is $O(k n)$, since we compute it for at most $O(n)$ pairs of clusters (see Section 3.3). Together with Lemmas 3.1 and 5.2, we obtain the following theorem:

Theorem 5.5. Let $S$ be a set of $n$ points in the Euclidean plane. Given the Voronoi diagram of $S$, the above method computes an $\varepsilon$-approximation of a complete linkage hierarchy of $S$ in $O(n / \sqrt{\varepsilon})$ time.

However, for very small values on $\varepsilon$ we can use the ideas from Section 4 to compute the $k$-distances in total time $O\left(n \log ^{2} k\right)$. Indeed, we only need to impose the following two modifications on the algorithm in that section:

1. Clean-up. Whenever a cluster is created such that the total sum of cardinalities of its vorchildren is $\geq 2 k$, replace its vorchildren by a single vorchild that consists of the $k$-extremes of the cluster (insert the new vorchild into the list of vorchildren and remove the old ones). 
2. Define the vorcardinality of a cluster as the total sum of cardinalities of its vorchildren, and use the vorcardinality instead of the actual cardinality throughout the algorithm (this affects only the merging of vorchildren).

By the clean-up operation we get that each cluster has at most $O(\log k)$ vorchildren, and that each of its $k$-extremes belongs to one of its vorchildren. To compute the $k$ distance between two clusters we use their vorchildren in the same way as in Section 4.

Let us calculate the time used for computing Voronoi structures. For simplicity, suppose that each point initially receives $\lceil 8.4 \log 2 k\rceil+2$ bank notes, each note having a value so that a point can participate in one clean-up or one merging of vorchildren (thus a bank note has value $O(\log k)$ since every vorchild has now cardinality at most $2 k)$. Let $v$ be an arbitrary point. When $v$ participates in a merging of vorchildren, either the new resulting vorchild (that $v$ becomes a member of) has cardinality at least 1.25 times greater than its previous vorchild, or its previous vorchild belongs to a cluster being merged with a cluster of at least the same vorcardinality. Thus $v$ cannot participate in more than $\lceil 4.2 \log 2 k\rceil$ mergings of vorchildren without participating in a clean-up. Further, when $v$ participates in a clean-up, at least $k$ points participating in this clean-up will never again belong to some vorchild. Therefore, if $v$ is not one of these points, $v$ can receive $\lceil 4.2 \log 2 k\rceil+1$ more bank notes from one of them. In this way, $v$ has enough bank notes to participate in additional $\lceil 4.2 \log 2 k\rceil$ mergings of vorchildren and one clean-up. Hence, we can conclude that the total time used for computing Voronoi structures is $O\left(n \log ^{2} k\right)$.

It remains to consider the time used for computing $k$-distances between clusters. Recall that every vorchild has now cardinality at most $2 k$. Therefore, referring to the proof of Theorem 4.1, we can use an analogous analysis but having $O(\log k)$ slots in arrays $Q$ and $E$ and using $O(\log k)$ coins when making a query. In this way, we can show that the total time used for computing $k$-distances is $O\left(n \log ^{2} k\right)$. Thus we obtain the following theorem:

Theorem 5.6. Let $S$ be a set of $n$ points in the Euclidean plane. Given the Voronoi diagram of $S$, an $\varepsilon$-approximation of a complete linkage hierarchy of $S$ can be computed in $O\left(n \log ^{2}(1 / \varepsilon)\right)$ time.

\section{Final Remarks and Further Research}

The techniques in this paper can also be adapted for other measures of proximity between two clusters, for example:

1. the perimeter of the convex hull (or of the smallest enclosing rectangle, circle, etc.) of their union; and

2. different sorts of weighted distances between the clusters (like the distance between their centroids).

For several such measures we can prove the analogue of Lemma 2.5, so the same approach yields fast algorithms. 
In addition, extending the approach to more than two dimensions, Lemmas 2.5 and 5.3 can still be adapted, by increasing the number of the so-called "triggers" and "types" of clusters used in their proofs (although the constants grow with the number of dimensions). It would also be interesting to prove Lemma 2.5 using a constant that is reasonably close to the true one.

\section{Acknowledgments}

We thank the anonymous referees for their valuable suggestions, and Dr. Günter Rote for his very helpful and encouraging comments, and for pointing out some errors in an earlier draft.

\section{References}

1. F. Aurenhammer. Voronoi diagrams-A survey of a fundamental geometric data structure. ACM Computing Surveys, 23(3):345-405, 1991.

2. F. Aurenhammer and R. Klein. Voronoi diagrams. Technical Report 198-5, Informatik, Fern Universität, Hagen, Germany, 1996.

3. W. H. E. Day and H. Edelsbrunner. Efficient algorithms for agglomerative hierarchical clustering methods. Journal of Classification, 1(1):7-24, 1984.

4. D. Defays. An efficient algorithm for a complete link method. Computer Journal, 20:364-366, 1977.

5. A. D. Gordon. Classification. Chapman and Hall, London, 1981.

6. A. K. Jain and R. C. Dubes. Algorithms for Clustering Data. Prentice-Hall, Englewood Cliffs, NJ, 1988.

7. D. G. Kirkpatrick. Optimal search in planar subdivisions. SIAM Journal on Computing, 12(1):28-35, 1983.

8. D. Krznaric and C. Levcopoulos. Computing a threaded quadtree (with links between neighbors) from the Delaunay triangulation in linear time. Proceedings of the 7 th Canadian Conference on Computational Geometry, pp. 187-192, 1995.

9. D. Krznaric and C. Levcopoulos. Computing hierarchies of clusters from the Euclidean minimum spanning tree in linear time. Proceedings of the 15th Conference on Foundations of Software Technology and Theoretical Computer Science, Lecture Notes in Computer Science, vol. 1026, pp. 443-455. SpringerVerlag, New York, 1995.

10. T. Kurita. An efficient agglomerative clustering algorithm using a heap. Pattern Recognition, 24(3):205209, 1991.

11. Křivánek. Connected admissible hierarchical clustering. Paper presented at the DIANA III Conference, Bechyne, Czechoslovakia, June 1990.

12. X. Li. Parallel algorithms for hierarchical clustering and cluster validity. IEEE Transactions on Pattern Analysis and Machine Intelligence, 12(11):1088-1092, 1990.

13. F. Murtagh. Complexities of hierarchic clustering algorithms: State of the art. Computational Statistics Quarterly, 1:101-113, 1984.

14. M. H. Overmars and J. van Leeuwen. Some principles for dynamizing decomposable searching problems. Information Processing Letters, 12(1):49-54, 1981.

15. F. P. Preparata and M. I. Shamos. Computational Geometry: An Introduction. Springer-Verlag, New York, 1985.

16. M. I. Shamos. Computational Geometry. Ph.D. thesis, Yale University, New Haven, CT, 1978.

Received September 18, 1995, and in revised form April 17, 1997. 